\title{
Molecular Tagging Velocimetry (MTV) and Its Automotive Applications
}

\author{
M. M. Koochesfahani, A. C. Goh, and H. J. Schock \\ Automotive Research Experiment Station (ARES) \\ Department of Mechanical Engineering \\ Michigan State University, East Lansing, MI 48824
}

\begin{abstract}
.
This work provides an overview of the technique of Molecular Tagging Velocimetry (MTV) and some of its automotive applications. The various elements of MTV implementation are briefly described in terms of the available molecular tracers, methods of tagging, detection, and processing schemes. The automotive applications of this velocimetry technique are demonstrated in mapping the velocity field of the intake flow into a "steady flow rig" model of an internal combustion engine and flow mapping of cycle-to-cycle variation in late compression of a motored IC engine.
\end{abstract}

\section{Introduction}

Molecular Tagging Velocimetry (MTV) is a whole field optical technique which relies on molecules that can be turned into long lifetime tracers upon excitation by photons of an appropriate wavelength. Typically a pulsed laser is used to "tag" the regions of interest, and those tagged regions are interrogated at two successive times within the lifetime of the tracer. The measured Lagrangian displacement vector provides the estimate of the velocity vector. This technique can be thought of as essentially a molecular counterpart of Particle Image Velocimetry (PIV), and it offers advantages compared to particle-based techniques where the use of seed particles is not desirable, difficult, or may lead to complications. Figure 1 illustrates one implementation of the technique where a planar grid of intersecting laser beams, formed from a pulsed excimer laser at $308 \mathrm{~nm}$, is used to turn on the luminescence of water-soluble supramolecules that are premixed in a water flow of a vortex ring approaching a solid wall at normal incidence (Gendrich et al. 1997). The displacement of the tagged regions is determined using a direct spatial correlation method. The conventional planar imaging shown in Figure 1 provides information on 
two components of the velocity vector, the projection onto the viewed plane. Stereo imaging can produce the complete three components of the velocity vector (Bohl et al. 2001).

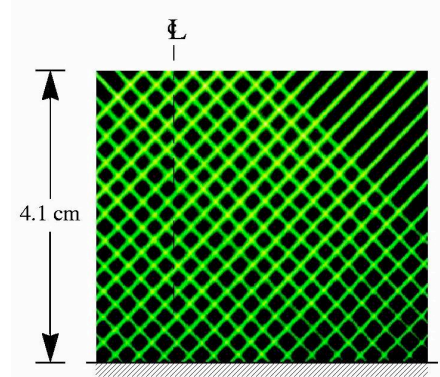

(a)

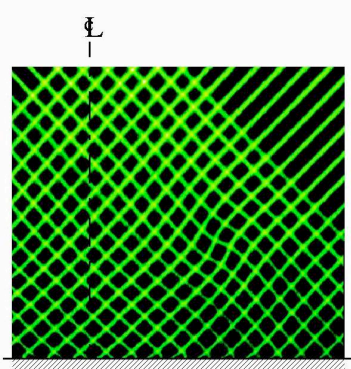

(b)

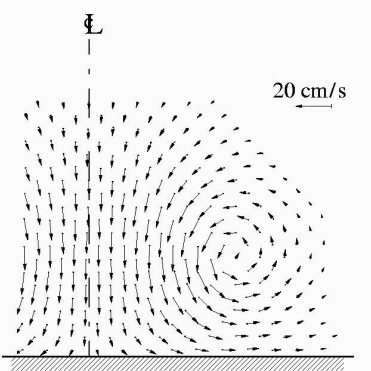

(c)

Fig. 1. Typical MTV image pairs and the resultant velocity field (Gendrich et al. 1997). The flow shown is from a vortex ring impacting on a flat wall at normal incidence. The axis of symmetry is indicated by the dashed lines. (a) The grid imaged $1 \square s$ after the laser pulse. (b) The same grid imaged 8 ms later. (c) The velocity field derived from (a) and (b).

The MTV technique has advanced significantly over the past decade in terms of the availability of new molecular tracers, methods of tagging, detection/imaging, and data processing. This paper provides only a brief description of such details. More complete reviews, along with more extensive list of related references and applications, can be found in Koochesfahani et al. (1996) and Koochesfahani (1999, 2000).

\section{Molecular Tracers, Tagging Methods, Detection, and Processing}

A molecular complex is suitable for molecular tagging applications if its lifetime as a tracer is long enough relative to the flow convection time scale to allow sufficient displacement of the tagged regions. The photophysics of the tracer, in turn, dictates the type and number of photon sources needed for tagging and interrogation.

The molecular tracers used to date in liquid-phase applications have most often been based on photochromic molecules in organic solvents (Popovich \& Hummel 1967; Falco \& Chu 1987), water-soluble caged fluorescent compounds (Lempert et al. 1995), and specially engineered water-soluble phosphorescent supramolecules (Gendrich et al. 1997). Since none of these tracers is naturally present in the flowing media where they are typically used, these molecules are first premixed in the flowing liquid. For gas-phase applications, techniques have been developed based on the use of excited-state oxygen (Miles et al. 1987, 1989), ozone $\mathrm{O}_{3}$ (Pitz et al. 1996; Ribarov et al. 1999), OH (Wehrmeyer et al. 1999), nitric oxide NO (Sijtsema et al. 2001; van der Laan 
et al. 2003), photodissociation of tert-butylnitrite (Krüger \& Grünefeld 1999), and phosphorescent molecules such as biacetyl (Stier \& Koochesfahani 1999) and acetone (Lempert et al. 2001). The first four of these tracers are generated from species naturally present in air, i.e. oxygen and water vapor in humid air or that generated as a result of combustion. As a result, their use is often referred to as "unseeded" applications. By contrast, the last three tracers have to first be seeded into the flowing gas stream. All the gas-phase flow examples given in Section 3 of this paper take advantage of biacetyl's phosphorescence.

Tagging along single or multiple lines is perhaps the simplest method of tagging and has been utilized in a large fraction of studies to date. An example of line tagging is shown in Figure 2, illustrating the trailing edge region of a NACA-0012 airfoil oscillating sinusoidally at a high reduced frequency of $k=$ 8.8 and amplitude of 2 degrees (Koochesfahani \& Bohl 2002). It is clear that line tagging allows the measurement of only one component of velocity, that normal to the tagged line. In addition, the estimate of this velocity component has an inherent error associated with it, which is connected with the ambiguity in the unique determination of the displacements of various portions of a (continuous) tagged line. In order to unambiguously measure two components of the velocity in a plane, the luminescence intensity field from a tagged region must have spatial gradients in two, preferably orthogonal, directions. For single-point velocimetry, this is easily achieved using a pair of crossing laser beams; a grid of intersecting laser lines allows multi-point velocity measurements as shown in Figure 1.
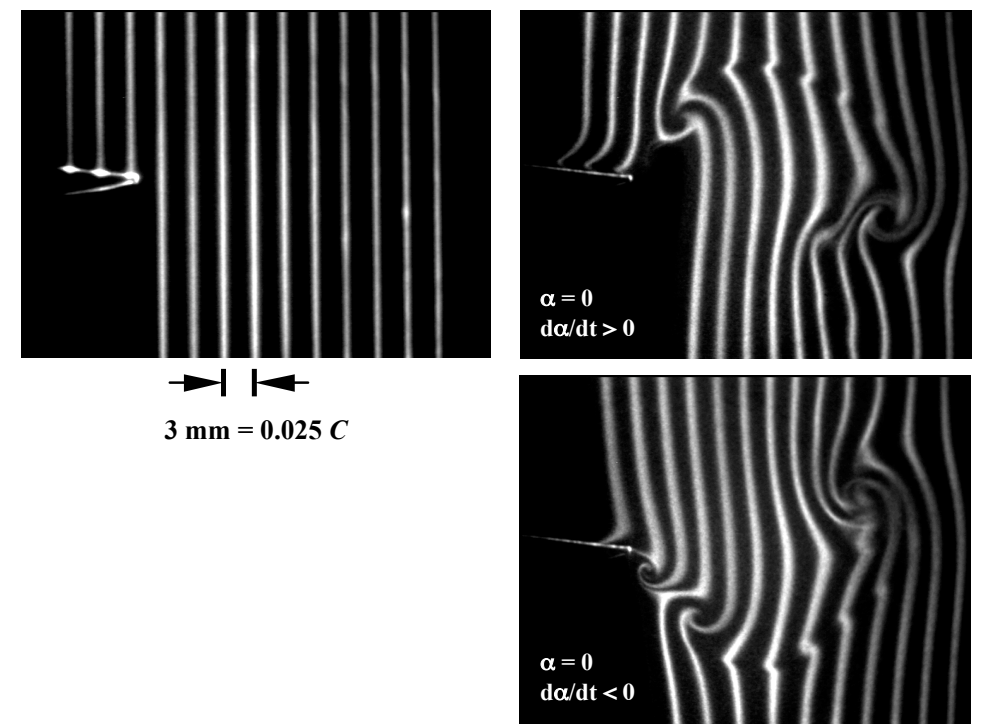

Fig. 2. Multi-line tagging in the trailing edge region of an oscillating NACA-0012 airfoil. The tagging pattern right after the laser pulse is a series of parallel lines with a spacing of $3 \mathrm{~mm}$ or about 0.025 chord length.

The MTV image pairs are acquired by a pair of CCD detectors that view the same region of interest in the flow through a beam splitter. Using a refer- 
ence target, the two cameras are aligned to within one pixel, and the remaining residual displacement field between the two detectors is quantified to sub-pixel accuracy and accounted for in subsequent data processing (for details see Gendrich et al. 1997). Immediately after the pulsed laser fires, the first detector records an initial image of the tagged regions. After a prescribed time delay, the second detector records a second image of the tagged regions displaced by the flow. As described in Gendrich et al. (1997), and Koochesfahani (1999), such a two-image system offers advantages over the typical single-image system in that no assumption needs to be made a priori about the intensity field in a tagged region. For example, one can properly take into account the variations in the initial tagging pattern (e.g. due to laser beam pointing instability, vibration of the optics, non-uniform tracer concentration, etc.), which could otherwise be misinterpreted as flow velocity fluctuations. In situations where the initial tagging pattern remains spatially invariant throughout the experiment, a single detector is sufficient; the initial (or reference) tagging pattern is recorded once, usually at the beginning of the experiment, and then the "delayed" images are acquired. In our work in liquid-phase flows, we use both nonintensified frame transfer cameras and gated image-intensified detectors, depending on the imaging requirements (e.g. field of view, time between image pairs). In gas-phase applications, we use gated image-intensified detectors. See Koochesfahani (1999) for several examples of applications in liquid and gas phase flows. These detectors are nominally $512 \times 512$ pixel arrays operating at 30 or 60 frame/s. The images are typically digitized to 8 bits by two image acquisition/ processing systems and transferred onto high capacity disk arrays in real time.

A common method for finding the displacement of tagged lines or grids has been to locate the center of each line through various techniques. Most of the recent techniques use the best fit to an assumed laser line shape, for example, a gaussian intensity distribution. A recent study (Hill \& Klewicki 1996) gives the accuracy in determining the displacement vector to be \pm 0.35 pixel rms. The performance of this method will suffer when the intensity distribution of the tagged regions cannot be assumed in advance, for example, due to nonuniform tracer distribution, difficulties associated with laser beam transmission through a flowing medium, bleaching effects, etc.

Our approach for finding the displacement of tagged regions is based on a direct digital spatial correlation technique, and offers certain advantages over the traditional line-center methods. In particular, it is a more general scheme that is independent of the specific intensity distribution within a tagged region and can accommodate arbitrary tagging patterns including those due to nonuniform scalar mixing fields. The details of this approach and its performance are described in Gendrich \& Koochesfahani (1996). A small window, referred to as the source window, is selected from a tagged region in the earlier image, and it is spatially correlated with a larger roam window in the second image. A well-defined correlation peak occurs at the location corresponding to the displacement of the tagged region by the flow; the displacement peak is located to sub-pixel accuracy using a multi-dimensional polynomial fit. Based on both experiments and an extensive statistical study, it has been found that the displacement of the tagged regions can be typically determined with a $95 \%$ con- 
fidence limit of \pm 0.1 sub-pixel accuracy (i.e. $95 \%$ of the displacement measurements are accurate to better than 0.1 pixel). This corresponds to an rms accuracy of \pm 0.05 pixel, assuming a Gaussian distribution for error. For high values of image $\mathrm{S} / \mathrm{N}$, the $95 \%$ confidence level can be as low as 0.015 pixel (0.0075 pixel rms). An example of the application of this procedure is provided in Figure 1; the velocity vectors shown in this figure are "raw" and have not been filtered or smoothed.

\section{Examples of MTV Measurements}

This section describes two recent automotive applications of MTV. Earlier applications of this technique in an IC engine geometry include the first multipoint velocity data in a two-stroke engine (Hilbert \& Falco 1991) and measurement of planar flow of consecutive cycles in a motored IC engine (Hascher et al. 1998). Many more examples of other flows can be found in Koochesfahani et al. (1996) and Koochesfahani (1999, 2000). These examples include, among others, boundary layer resolved measurements of unsteady separation on pitching airfoils (Gendrich 1998), vortex flows and mixing enhancement (Cohn 1999), three-dimensional vortex core structure (Bohl 2002), and buoyancy-driven convective flow in directional solidification (Wirtz et al. 1998; Lum et al. 2001). In addition to purely velocimetry applications, recent studies are extending MTV to multi-variable mapping such as simultaneous velocity-concentration (Koochesfahani et al. 2000) and velocity-temperature (Thomson \& Maynes 2001; Hu \& Koochesfahani 2003).

In the examples that follow, the phosphorescent tracer biacetyl is used for MTV (Stier \& Koochesfahani 1999). In this case, only one photon source is needed, the tagging process occurs during the brief laser pulse and the long lifetime tracer is the excited state molecule itself. However, because biaceyl's phosphorescence is effectively quenched by oxygen, its use is limited to oxygen-free environments (typically $\mathrm{N}_{2}$ is used). In our work, a Lambda Physik $\mathrm{XeCl}$ excimer laser $(\square=308 \mathrm{~nm}, 20 \mathrm{~ns}$ pulse) is used as the excitation source. Detection is achieved by Xybion (ISG-350-GW3) gated image-intensified cameras, operating at $30 \mathrm{frame} / \mathrm{s}$.

\subsection{Measurements in a "Steady Flow Rig" Model of an IC Engine}

The steady flow rig configuration is commonly used in the IC engine research community to study the fundamentals of the intake flow. The particular geometry used consists of a quartz cylinder of radius $R_{o}=41 \mathrm{~mm}$, placed axisymmetrically around a nozzle with a valve body placed axisymmetrically inside the jet nozzle. In this case the flow exiting through the valve opening, which simulates the intake flow into an IC engine geometry, is in the form of an annular jet. In this study, the valve opening (valve lift) is set at $\mathrm{L}=9 \mathrm{~mm}$ and the maximum intake speed is about $10 \mathrm{~m} / \mathrm{s}$. The instantaneous accelera- 
tions in the shear layer at the interface between the intake jet and adjacent fluid can be as high as $5000 \mathrm{~g}$, making it difficult to rely on the results of particle-based techniques. The details of this work can be found in Stier \& Koochesfahani (1999).

Figure 3 shows a $3 \mathrm{~cm} \times 3 \mathrm{~cm}$ field of view in the nitrogen/biacetyl flow being investigated and the regions tagged by a grid pattern. Part of the valve body and the left wall of the cylinder are visible in the picture. The maximum flow speed in the annular jet entering the cylinder is about $10 \mathrm{~m} / \mathrm{s}$. Also shown is an example of the later image of the tagged regions after a $50 \mu$ delay. For this time delay, the maximum displacement of tagged regions is about 8 pixels $(\sim 500 \mu \mathrm{m})$. Image pairs such as those in Figure 3 are used to determine the instantaneous radial and axial velocity components in this flow field. An example of the instantaneous velocity field and the structure of the intake flow in this geometry are shown in Figure 4 along with the average velocity field based on 320 realizations. The instantaneous flow map shows a highly unsteady intake annular jet, which as has an undulating appearance with opposite sign large scale vortical structures on its two sides. The mean flow map indicates a large scale region of recirculation in the upper left corner of the engine cylinder, a feature typical of an IC engine flow field. These data have also been used to derive other properties of the flow such as the instantaneous and average vorticity fields and velocity fluctuations (Stier \& Koochesfahani 1999).

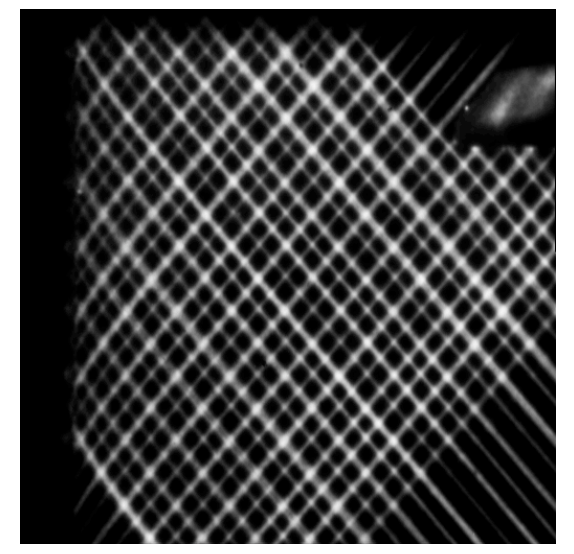

Tagged regions right after the laser pulse

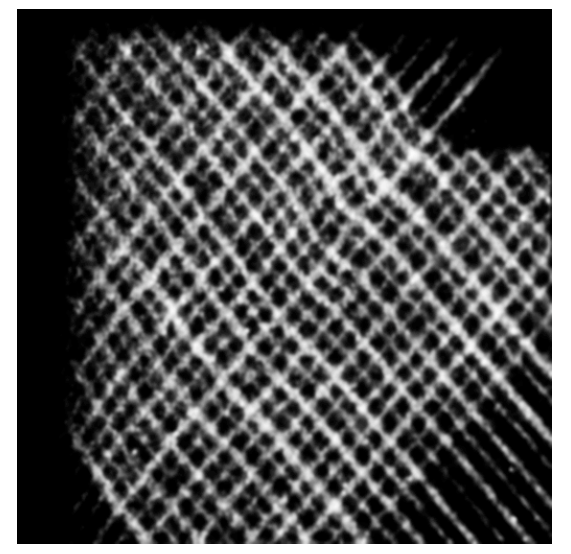

Tagged regions $50 \square \mathrm{s}$ later; $3 \mathrm{~cm} \times 3 \mathrm{~cm}$ field of view

Fig. 3. Example of the tagging pattern for the gas flow into the steady flow rig (Stier \& Koochesfahani 1999). 


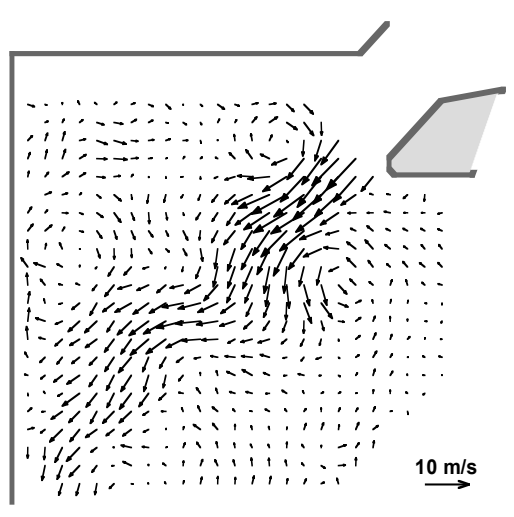

The instantaneous velocity field derived from the image pair in Figure 3 using a spatial correlation procedure.

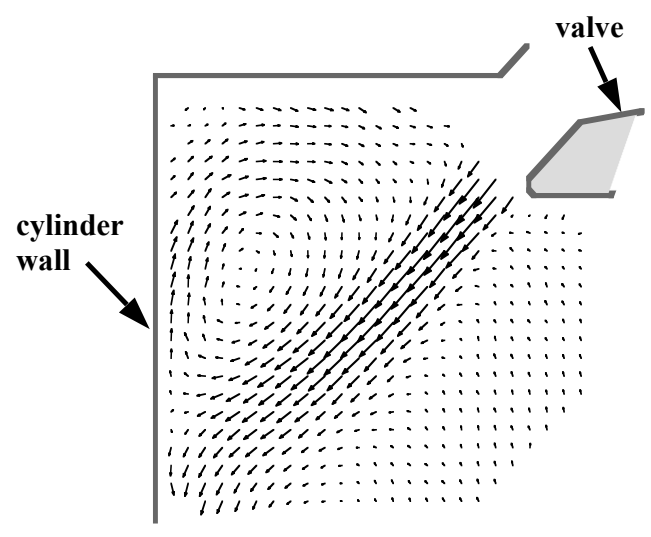

Time-averaged velocity field based on 320 instantaneous realizations.

Fig. 4. Intake flow into a steady flow rig model of IC engine measured with MTV using nitrogen seeded with biacetyl (Stier \& Koochesfahani 1999).

\subsection{Velocity Field during Late Compression in a Motored IC Engine}

One of the main obstacles in optimizing combustion in gasoline-fueled internal combustion engines is the large cycle-to-cycle variation in in-cylinder flow and mixing characteristics. Cycle-to-cycle variability puts constraints on the lean limits of combustion. In this study velocity field data were obtained using MTV during late compression of an internal combustion engine, the most critical time of the four-stroke cycle. Such data are highly sought since the state of the flow just before the firing of the spark plug directly influences the subsequent combustion and emission production.

The measurements are conducted in an optically accessible motored research engine, see Figure 5. The engine specifications are given in Table 1. The cylinder is made from quartz and the flat-head piston face is modified for optical access through a quartz window. A typical MTV grid generated within the engine cylinder is also shown in Figure 5. Measurements are made at late compression at a crank angle of $270 \mathrm{CAD}$, as the piston approaches the Top Dead Center (TDC) of the engine, with the engine running at 600 RPM. For the conditions described here, and the engine compression ratio of nearly 10 , the gas temperature can reach a value as high as $600 \mathrm{~K}$. The measurements consist of 500 independent realizations of the velocity map at the same crack angle $(270 \mathrm{CAD})$ at the mid-tumble-plane. The position of the imaged plane over which the velocity maps are obtained is given in Figure 6. Further details of this work can be found in Goh (2001). 
Table 1. Engine specification

$\begin{array}{ll}\text { Model and Make } & \text { Ford 4-Valve 4.6L } \\ \text { Bore and Stroke } & 90.2 \mathrm{~mm} / 90.0 \mathrm{~mm} \\ \text { Connecting Rod Length } & 150.7 \mathrm{~mm} \\ \text { Valve Activation } & \text { DOHC } \\ \text { Intake Valve Diameter } & 37.0 \mathrm{~mm} \\ \text { Exhaust Valve Diameter } & 30.0 \mathrm{~mm} \\ \text { Maximum Valve Lift } & 10.02 \mathrm{~mm} \text { at 120 CAD } \\ \text { Zero CAD Intake TDC } & \text { Intake TDC } \\ \text { Intake Valve Opening } & \text { 6 CAD Before TDC } \\ \text { Intake Valve Closure } & \text { 250 CAD After BDC } \\ \text { Exhaust Valve Opening } & \text { 126 CAD After TDC } \\ \text { Exhaust Valve Closure } & \text { 16 CAD After TDC } \\ \text { Compression Ratio } & 9.85: 1 \\ \text { Piston Top } & \text { Flat }\end{array}$

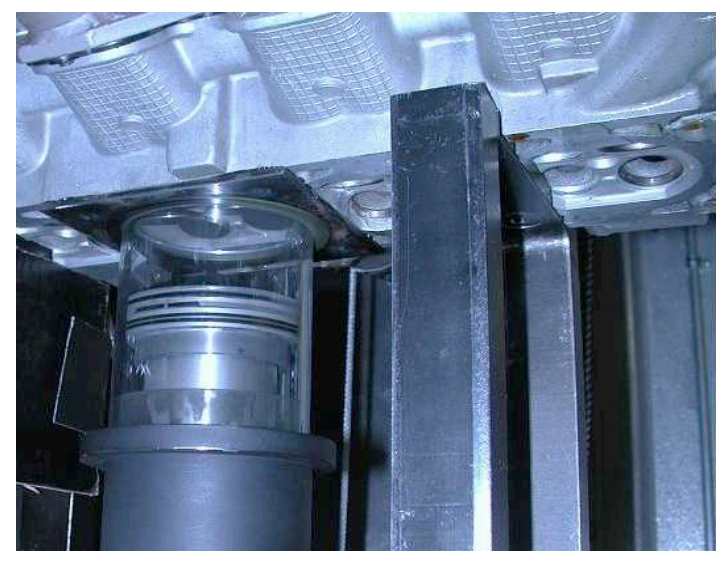

Optically accessible Ford 4-valve, 4.6L engine

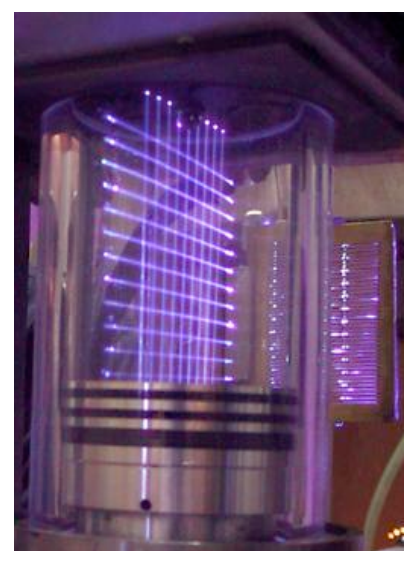

Sample MTV grid

Fig. 5. The optically accessible research engine and a typical MTV grid tagging the fluid within the engine cylinder. 


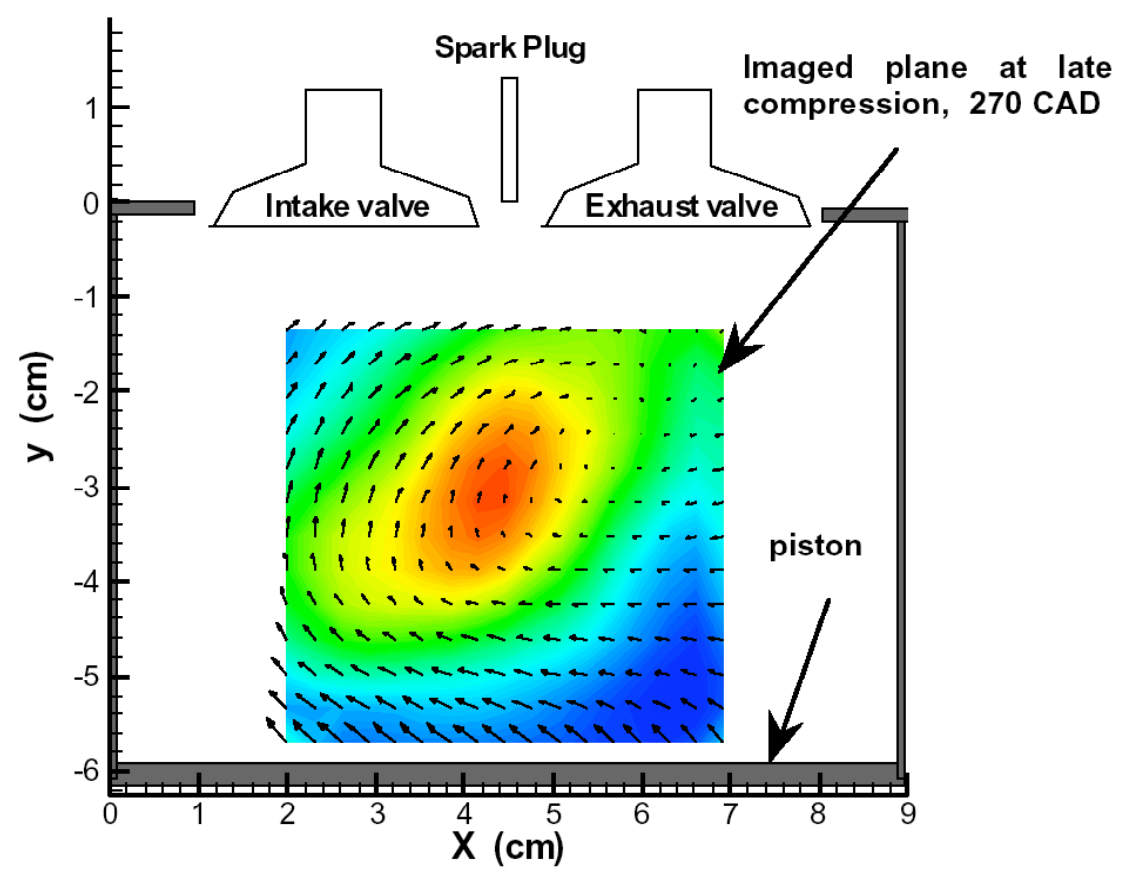

Fig. 6. The position of the imaged plane at $270 \mathrm{CAD}$.

Two samples of the instantaneous velocity field, for two different engine cycles, are shown in Figure 7. It is clear the flow pattern is significantly different between these two realizations, an indication of the large cycle-to-cycle variability of the flow. The maps of the ensemble-averaged velocity field and the rms fluctuation of the horizontal velocity component are computed from 500 such realizations, see Figure 8. As expected for a flow with large cycle-to-cycle variation, the mean velocity field has little resemblance to the instantaneous field; the local fluctuation level can be higher than the mean by several hundred percent. The data shown in Figures 7 and 8 are the first cycle-resolved velocity measurements that use molecular tracers rather than particles to determine flow velocities in a piston-engine assembly. Such measurements enabled by the MTV imaging technique are being used to advance the understanding and quantification of cycle-to-cycle variability in an internal combustion engine, and finding methods for reducing it (Goh 2001). 

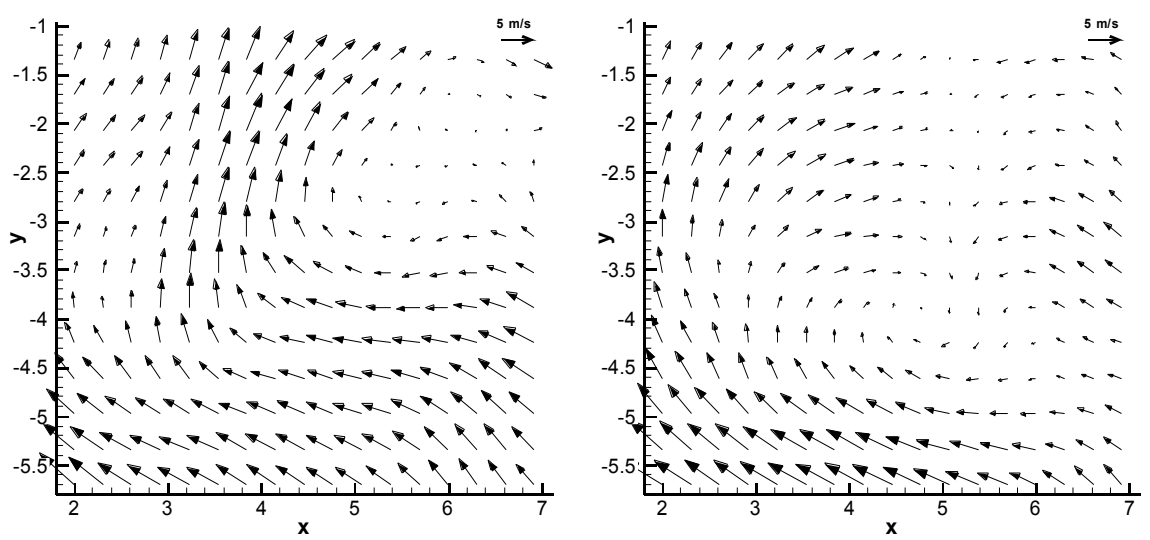

Fig. 7. Two instantaneous realizations at $270 \mathrm{CAD}$ showing the large cycle-to-cycle variability of the flow field.

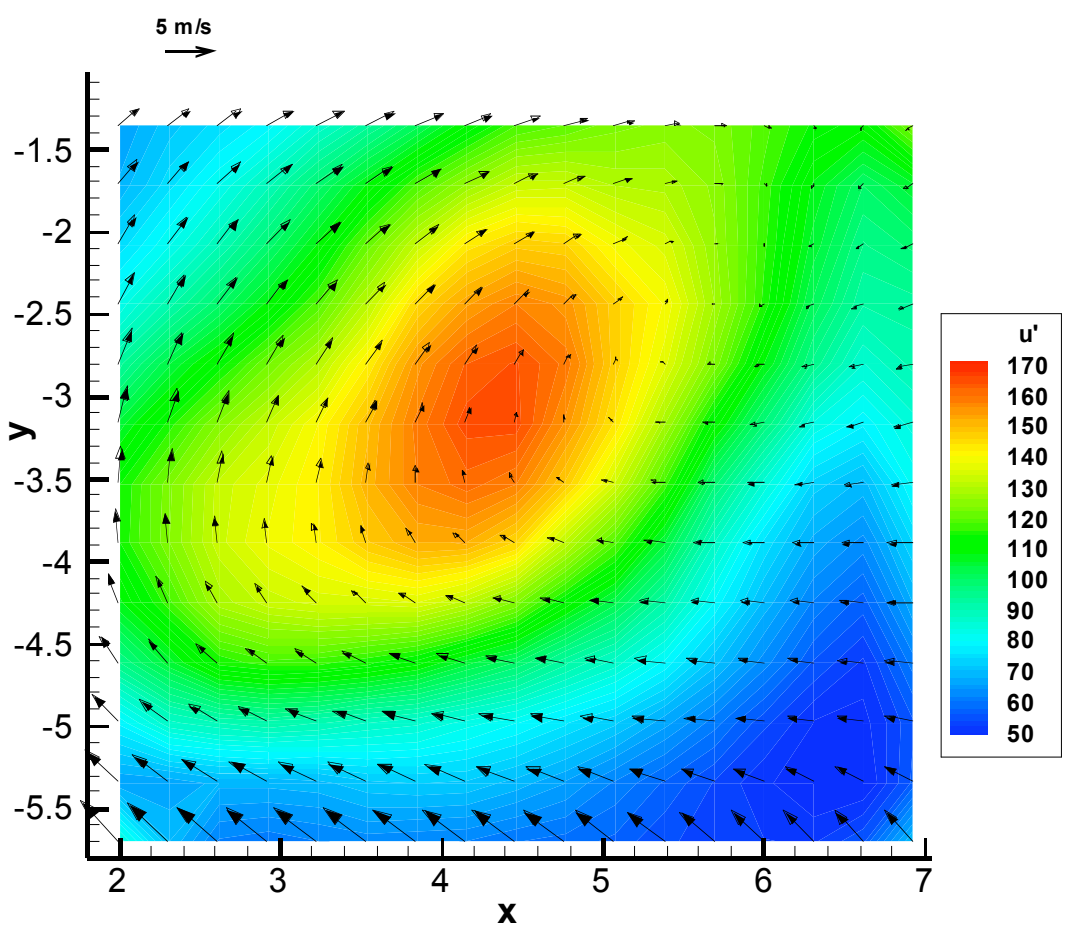

Fig. 8. Maps of the ensemble-averaged velocity field and the rms fluctuation of the horizontal component of velocity u' (in $\mathrm{cm} / \mathrm{s}$ ). Colors denote rms fluctuation level. 


\section{Conclusions}

A brief overview of the Molecular Tagging Velocimetry (MTV) technique is given, along with two of its automotive applications. These applications include mapping the velocity field of the intake flow into a "steady flow rig" model of an internal combustion engine and flow mapping of cycle-to-cycle variation in late compression of a motored IC engine. The MTV approach has continuously evolved and is now being utilized as a tool in both fundamental flow studies and applied engineering measurements.

\section{Acknowledgments}

This work was supported by the MRSEC Program of the National Science Foundation, Award Numbers DMR-9400417 and DMR-9809688, Department of Energy Grant No. DE-FC02-99EE50574, and Ford Motor Company.

\section{References}

Bohl, D., and Koochesfahani, M., and Olson, B. (2001) Development of stereoscopic Molecular Tagging Velocimetry," Exp. Fluids, vol. 30, 302308.

Bohl, D. G. (2002) Experimental Study of the 2-D and 3-D Structure of a Concentrated Line Vortex Array, PhD thesis, Michigan State University.

Cohn, R. K. (1999) Effect of forcing on the vorticity field in a confined wake, $\mathrm{PhD}$ thesis, Michigan State University.

Falco, R. E. and Chu, C. C. (1987) Measurement of two-dimensional fluid dynamic quantities using a photochromic grid tracing technique, SPIE, vol. 814, 706-710.

Gendrich, C. P. and Koochesfahani, M. M. (1996) A spatial correlation technique for estimating velocity fields using Molecular Tagging Velocimetry (MTV), Exp. Fluids, vol. 22, no. 1, 67-77.

Gendrich, C. P., Koochesfahani, M. M. and Nocera, D. G. (1997) Molecular tagging velocimetry and other novel applications of a new phosphorescent supramolecule, Exp. Fluids, vol. 23, no. 5, 361-372.

Gendrich, C. P. (1998) Dynamic stall of rapidly pitching airfoils: MTV experiments and Navier-Stokes simulations, PhD thesis, Michigan State University.

Goh, A. C. H. (2001) Active flow control for maximizing performance of spark-ignited stratified charge engines, MS thesis, Michigan State University.

Hascher, H. G., Novak, M., Lee, K., Schock, H., Rezaei, H., and Koochesfahani, M. M. (1998) An evaluation of IC-engine flow with the use of modern in-cylinder measuring techniques, AIAA Paper 98-3455. 
Hill, R. B. and Klewicki, J. C. (1996) Data reduction methods for flow tagging velocity measurements, Exp. Fluids, vol. 20, no. 3, 142-152.

Hilbert, H. S. and Falco, R. E. (1991) Measurements of flows during scavenging in a two-stroke engine, SAE Technical Paper 910671.

$\mathrm{Hu}, \mathrm{H}$. and Koochesfahani, M. M. (2003) A novel technique for quantitative temperature mapping in liquid by measuring the lifetime of laser induced phosphorescence, Journal of Visualization, vol. 6, no. 2, 143-153.

Koochesfahani, M. M., Cohn, R. K., Gendrich, C. P. and Nocera, D. G. (1996) Molecular tagging diagnostics for the study of kinematics and mixing in liquid phase flows, Proceedings of the Eighth International Symposium on Applications of Laser Techniques to Fluids Mechanics, July 8 - 11, 1996, Lisbon, Portugal, vol. I, 1.2.1-1.2.12; Also in Developments in Laser Techniques and Fluid Mechanics, Chapter 2, section 1, p. 125, Eds. Adrian, Durao, Durst, Maeda, and Whitelaw; Springer-Verlag, Berlin, 1997.

Koochesfahani, M. M. (1999) Molecular Tagging Velocimetry (MTV): Progress and Applications, AIAA Paper 99-3786.

Koochesfahani, M. M. (Ed) (2000) Special Feature: Molecular Tagging Velocimetry, Meas. Sci. Technol., vol. 11, no. 9, 1235-1300.

Koochesfahani, M., Cohn, R, and MacKinnon, C. (2000) Simultaneous whole-field measurements of velocity and concentration fields using combined MTV and LIF, Meas. Sci. Technol., vol. 11, no. 9, 1289- 1300.

Koochesfahani, M. M. and Bohl, D. (2002) Molecular tagging visualization and velocimetry of the flow at the trailing edge of an oscillating airfoil," Proceedings of the $10^{\text {th }}$ International Symposium on Flow Visualization, Kyoto, Japan, Paper No. F0453.

Krüger, S. and Grünefeld, G. (1999) Stereoscopic flow-tagging velocimetry, Appl. Phys. B, vol. 69, 509-512.

Lempert, W. R., Magee, K., Ronney, P., Gee, K. R., and Haughland, R. P. (1995) Flow tagging velocimetry in incompressible flow using photoactivated nonintrusive tracking of molecular motion (PHANTOMM), Exp. Fluids, vol. 18, 249-257.

Lempert, W. R., Jiang, N., Sethuram, S., and Samimy, M. (2001) Molecular tagging velocimetry measurements in supersonic micro nozzles, AIAA Paper 2001-0244.

Lum, C, Koochesfahani, M. M., and McGrath, J. J. (2001) Measurements of the velocity field with MTV during the solidification of an alloy analog with mushy region, ASME/IMECE2001 Paper HTD-24222.

Miles, R., Cohen, C., Conners, J., Howard, P., Huang, S., Markovitz, E., and Russell, G. (1987) Velocity measurements by vibrational tagging and fluorescent probing of oxygen, Optics Letters, vol. 12, no. 11, 861-863.

Miles, R. B., Connors, J. J., Markovitz, E. C., Howard, P. J., and Roth, G. J. (1989) Instantaneous profiles and turbulence statistics of supersonic free shear layers by Raman Excitation plus Laser-Induced Electronic Fluorescence (RELIEF) velocity tagging of oxygen, Exp. Fluids, vol. 8, 17-24.

Pitz, R. W., Brown, T. M., Nandula, S. P., Skaggs, P. A., DeBarber, P. A., Brown, M. S., and Segall, J. (1996) Unseeded velocity measurement by ozone tagging velocimetry, Optics Letters, vol. 21, no. 10, 755-757. 
Popovich, A. T. and Hummel, R. L. (1967) A new method for nondisturbing turbulent flow measurement very close to a wall, Chem. Eng. Soc., vol. 22, 21-25.

Ribarov, L. A., Wehrmeyer, J. A., Batliwala, F., Pitz, R. W., and DeBarber, P. A. (1999) Ozone tagging velocimetry using narrowband excimer lasers, AIAA J., vol. 37, no. 6, 708-714.

Sijtsema, N.M., Dam, N. J., Klein-Douwel, RJH, and ter Meulen, J.J. (2001) Molecular tagging velocimetry in unseeded air flows, AIAA Paper 20010851.

Stier, B. and Koochesfahani, M. M. (1999) Molecular tagging velocimetry (MTV) measurements in gas phase flows, Exp. Fluids, vol. 26, no. 4, 297304.

Thomson, S. L. and Maynes, D. (2001) Spatially resolved temperature measurements in a liquid using laser induced phosphorescence, J. Fluids Engr., vol. 123, 293-302.

Van der Laan, W.P.N., Tolboom, R. A. L., Dam, N.J., and ter Meulen, J.J. (2003) Molecular tagging velocimetry in the wake of an object in supersonic flow, Exp. Fluids, vol. 34, 531-533.

Wehrmeyer, J. A., Ribarov, L. A., Oguss, D. A., and Pitz, R. W. (1999) Flame flow tagging velocimetry with $193 \mathrm{~nm} \mathrm{H}_{2} \mathrm{O}$ photodissociation, $A p$ plied Optics, vol. 38, 6912-6917.

Wirtz, K., Koochesfahani, M. M., McGrath, J. J., and Benard, A. (1998) Molecular tagging velocimetry applied to buoyancy-driven convective phenomena during solidification, ASME Paper HTD-vol. 361-4, 103. 